\title{
Relationship between anthropometric characteristics and aerobic fitness among Malaysian men and women
}

\author{
Syazni Razak', Maria Justine ${ }^{1, *}$, Vikram Mohan² \\ ${ }^{1}$ Centre for Physiotherapy Studies, Faculty of Health Sciences, Universiti Teknologi MARA Selangor, Puncak Alam Campus, Selangor, Malaysia \\ ${ }^{2}$ Department of Rehabilitation and Sports Sciences, Faculty of Health and Social Science, Bournemouth University, Bournemouth, UK
}

This cross-sectional study evaluated the relationships between anthropometric and aerobic fitness (rate of perceived exertion [RPE] and predicted maximal oxygen uptake $\left[\mathrm{VO}_{2 \max }\right]$ ) among 228 participants (age: $23.78 \pm 4.42$ years). $\mathrm{RPE}$ and predicted $\mathrm{VO}_{2 \max }$ were determined during the cycle ergometer exercise test. Data were also obtained for height, weight, body mass index (BMI), hip and waist (WC) circumferences. Data analysis revealed $\mathrm{VO}_{\text {max }}$ is correlated with $\mathrm{WC}(r=-0.571)$, weight $(r=-0.521)$, waist-to-height ratio (WHtR) $(r=-0.516)$, waist-to-hip ratio (WHR) $(r=-0.487)$, and BMI $(r=-0.47)$ in men, while, in women with WC $(r=-0.581)$, weight $(r=-0.571)$, WHtR $(r=-0.545)$, BMI $(r=-0.545)$, WHR $(r=-0.473$ ), and height $(r=-0.287)$ (all $P<0.05)$. Regression analysis showed WC was a significant predictor for $\mathrm{VO}_{2 \max }$ in men and women $\left(r^{2}=32.6 \%\right.$ vs. $33.7 \%$ ). The receiver operating characteristic curve of WC showed 0.786 and 0.831 for men and women, respectively. WC or abdominal obesity is the strongest predictor for $\mathrm{VO}_{2 \max }$, which is an indicator of aerobic fitness in Malaysian adults.

Keywords: Aerobic fitness, Anthropometry, Malaysia, Submaximal cycle test, Waist circumference

\section{INTRODUCTION}

In Malaysia, a study by the National Health and Morbidity Survey (NHMS, 2019) reported that the prevalence of abdominal obesity has increased drastically from $30.6 \%$ to $52.6 \%$ and it is accounted that 1 in 2 Malaysians are overweight, obese or has abdominal obesity (NHMS, 2019). Alarmingly, the NHMS also reported that cardiovascular disease has become the number one killer in Malaysia, that could be due to physical inactivity, as 1 in 4 Malaysian adults was not physically active (NHMS, 2019). Physical inactivity and bad eating patterns are known factors of obesity (Amirabdollahian and Haghighatdoost, 2018). On the other hand, obesity and aerobic fitness have been established to affect the health outcome of cardiovascular diseases and mortality and are well-established predictors of metabolic syndromes (Amirabdollahian and Haghighatdoost, 2018).

Body mass index (BMI) has long been used as a proxy indicator of adiposity that predicts morbidity and mortality from several chronic diseases, such as diabetes, heart diseases, and stroke (Amirabdollahian and Haghighatdoost, 2018). However, studies have found that waist circumference (WC) is a stronger predictor than BMI, in which WC is a significant predictor of comorbidity and cardiorespiratory fitness (Janssen et al., 2004). While a few studies have conflicting conclusions on which anthropometric measures that best predicts aerobic fitness or risk of cardiovascular diseases (Ahmad et al., 2016; Alshamiri et al., 2020; Tran et al., 2018). We believed that measuring anthropometry parameters are more practical, however, findings from studies among the Malaysians are not well-established.

On the other hand, methods of assessing aerobic fitness also varied in terms of its practicality. For example, Oueslati et al. (2018) applied the Bruce protocol with a direct connection to an ergospirometer for gas analysis which is considered a gold standard to obtain $\mathrm{VO}_{2 \max }$ value, but it may be unsuitable for all population, especially untrained individuals. This is because the protocol has fixed speeds, and the inclination is not introduced gradually which
${ }^{\star}$ Corresponding author: Maria Justine (D) https://orcid.org/0000-0002-2412-8653 Centre for Physiotherapy Studies, Faculty of Health Sciences, Universiti Teknologi MARA Selangor, Puncak Alam Campus, 42300 Puncak Alam, Selangor, Malaysia Email: maria205@uitm.edu.my

Received: January 10, 2021 / Accepted: January 24, 2021
This is an Open Access article distributed under the terms of the Creative Commons Attribution Non-Commercial License (https://creativecommons.org/licenses/by-nc/4.0/) which permits unrestricted non-commercial use, distribution, and reproduction in any medium, provided the original work is properly cited. 
it occurs immediately at the start of each stage. Additionally, this test may induce stress that may be unsuitable for untrained individuals (Badawy and Muaidi, 2019). Another study reported that physically inactive and overweight individuals prefer the cycling test over the treadmill test because it is easier to perform (Loftin et al., 2004). However, the selection of suitable equipment depends on the availability of equipment, risk, type of participants, and objective of the test to be performed. Thus, the American College of Sports Medicine (ACSM, 2013) suggested the use of submaximal exercises, such as the Astrand-Rhyming cycle ergometer test, which is more feasible and suitable (Lennon et al., 2012). The Astrand-Rhyming cycle ergometer has many benefits; it allows for heart rate, blood pressure and electrocardiographic to be obtained easily, a nonweight bearing performance that is well tolerated by individuals that have physical restrictions as well as for healthy individuals (Lennon et al., 2012). Besides, this protocol also allows the measurement of the rate of perceived exertion (RPE) which is the subjective feeling of fatigue as it is associated with lactate level and muscular activity during exercise (Elsangedy et al., 2013).

The current study aimed to investigate which anthropometric parameters can best explains the influence on aerobic fitness (RPE and predicted $\left.\mathrm{VO}_{2 \max }\right)$ in physically inactive adults. The findings of this study may be useful for clinicians to apply a simple, quick, and practical method to assess one's health status.

\section{MATERIALS AND METHODS}

\section{Participants and study design}

This cross-sectional study was performed among students and staff of one of the largest government-funded universities in Malaysia. Participants included were: (a) age, 18-40 years old; (b) both men and women; (c) normal weight to obese $\left(\geq 18.5 \mathrm{~kg} / \mathrm{m}^{2}\right)$; (d) answered "no" to all the questions in the Physical Activity Readiness Questionnaire (PAR-Q); and (e) not involved in a moderateintensity exercise at least 3 times a week. Those excluded were (a) smokers; (b) diagnosed with chronic diseases, such as cardiovascular and neuromuscular conditions; and (c) underweight. The study protocol was approved by the Research Ethics Committee of Universiti Teknologi MARA (approval number: REC/290/18). All participants received detailed information about the objectives and methods of the study and provided informed consent forms. The study was conducted at the Exercise Testing Laboratory. The screening procedures required the participants to complete the demographic data, including age, gender, smoking status, medical history, and PAR-Q.

\section{Anthropometric characteristics}

A tape measure was used to measure $\mathrm{WC}$ and hip circumference (HC) to the nearest $0.1 \mathrm{~cm}$ in standing position. The landmark for measuring WC was around the abdomen, midway between the level of the lowest rib and the iliac crest on the horizontal plane. $\mathrm{HC}$ was measured by placing the tape measure at the widest part of the buttock. WC and $\mathrm{HC}$ were used to calculate the waist-tohip ratio (WHR) and waist-to-height ratio (WHtR), in which WC was divided by HC and height, respectively. Participants' height was measured using a stadiometer, while body weight and BMI were measured using a body composition analyzer.

\section{Predicted $\mathrm{VO}_{2 \max }$ and $\mathrm{RPE}$}

The guideline from ACSM was applied to conduct the submaximal cycle ergometer exercise test to predict the $\mathrm{VO}_{2 \max }$ which is the main predictor of physical performance and aerobic fitness (Borg, 1982). Participants underwent the test that lasted for $6 \mathrm{~min}$, and conducted between 9 a.m. till 12 noon. The pedal was set to $50 \mathrm{rpm}$, and the target heart rate was within the range of 125170 beats/min. Heart rate was measured by using a pulse oximeter at the 5th and 6th min of the test, in which the average heart rate was used to estimate $\mathrm{VO}_{2 \max }$ in accordance with the AstrandRhyming nomogram. A line was drawn on the Astrand-Rhyming nomogram from the average heart through the $\mathrm{VO}_{2 \max }$ and to the use of workload during the test, according to sex. The intersected line on $\mathrm{VO}_{2 \max }$ was used to predict the participants' maximal oxygen uptake. Age was adjusted with $\mathrm{VO}_{2 \max }$ by using a correction factor (Åstrand and Ryhming, 1954). The RPE was assessed using the Borg's 6-20 RPE scale (Borg, 1982). The participants were explained about Borg's RPE scale and reminded them to rate their exertion throughout the test. Prior to the exercise test, the participants were allowed to warm up for 2 min.

\section{Data analysis}

The data were analyzed using IBM SPSS Statistics ver. 22.0 (IBM Co., Armonk, NY, USA). The demographic data, WC, WHR, $\mathrm{WHtR}, \mathrm{RPE}$, and $\mathrm{VO}_{2 \max }$ of the participants were analyzed in terms of sex differences via an independent t-test. Pearson correlation test was used to determine the relationships of anthropometric parameters with $\mathrm{RPE}$ and $\mathrm{VO}_{2 \max }$. Stepwise multiple linear regression was conducted to determine the relationship of the variables of interest for both sexes. To perform multiple comparisons, only Pearson correlation that presented an $r$-value of at least 0.4 and $P<0.05$ were selected (Vancampfort et al., 2015). DurbinWatson and variation inflation factor tests were included for re- 
gression analysis to indicate autocorrelation and multicollinearity of the independent variables, respectively. A value below 2.0 indicates the absence of autocorrelation whereas a value below 10 indicates the absence of multicollinearity (Rehman and Dawood, 2017). The receiver operating characteristic (ROC) curve was performed to indicate the diagnostic sensitivity (true-positive rate) and specificity (true negative rate) across the range of possible predictive values for the independent variable. The level of significance was set at $P<0.05$.

\section{RESULTS}

\section{Characteristics of participants}

Table 1 presents a comparison of the characteristics of the participants $(\mathrm{N}=228)$ for this study. Men were significantly higher

Table 1. Characteristics of participants $(\mathrm{N}=228)$

\begin{tabular}{lcccl}
\hline Characteristic & $\begin{array}{c}\text { All } \\
(\mathrm{N}=228)\end{array}$ & $\begin{array}{c}\text { Men } \\
(\mathrm{n}=99)\end{array}$ & $\begin{array}{c}\text { Women } \\
(\mathrm{n}=129)\end{array}$ & $\begin{array}{l}P \text {-value } \\
\text { Age }(\mathrm{yr})\end{array}$ \\
$23.78 \pm 4.42$ & $24.22 \pm 4.99$ & $23.44 \pm 3.92$ & 0.201 \\
Weight $(\mathrm{kg})$ & $67.09 \pm 16.11$ & $71.41 \pm 13.86$ & $63.77 \pm 16.95$ & $0.001^{* *}$ \\
Height $(\mathrm{cm})$ & $161.82 \pm 8.2$ & $168.38 \pm 5.9$ & $156.79 \pm 5.8$ & $0.001^{* *}$ \\
Body mass index $\left(\mathrm{kg} / \mathrm{m}^{2}\right)$ & $25.12 \pm 4.75$ & $24.99 \pm 4.38$ & $25.21 \pm 5.03$ & 0.73 \\
Waist circumference $(\mathrm{cm})$ & $80.76 \pm 14.97$ & $85.61 \pm 12.11$ & $77.04 \pm 15.89$ & $0.001^{* *}$ \\
WHR & $0.8 \pm 0.08$ & $0.86 \pm 0.06$ & $0.75 \pm 0.06$ & $0.001^{* *}$ \\
WHtR & $0.5 \pm 0.09$ & $0.51 \pm 0.07$ & $0.49 \pm 0.1$ & 0.121 \\
RPE & $13.42 \pm 2.77$ & $13.82 \pm 2.88$ & $13.11 \pm 2.64$ & 0.054 \\
Predicted V0 2 max & $54.67 \pm 22.79$ & $37.38 \pm 9.79$ & $39.19 \pm 10.41$ & 0.179 \\
(mL/kg/min) & & & & \\
\hline
\end{tabular}

Values are presented as mean \pm standard deviation. Comparisons were tested using independent $t$-test.

WHR, waist-to-hip ratio; WHtR, waist-to-height ratio; RPE, rate perceive exertion; $\mathrm{VO}_{2 \max }$, maximal oxygen consumption.

${ }^{* *}$ The mean difference is significant at the level of $P<0.01$. in height, weight, WC and WHR compared to women (all, $P<$ $0.05)$. Men scored slightly higher $\mathrm{RPE}$ but lower $\mathrm{VO}_{2 \max }$ compared to women, but were not significantly different (both, $P<0.05$ ).

\section{Pearson correlation between anthropometric with RPE and aerobic fitness in men and women}

Table 2 presents the correlation results of RPE and predicted $\mathrm{VO}_{2 \max }$. Among men, no significant correlation was found in RPE with all the independent variables, however, predicted $\mathrm{VO}_{2 \max }$ was inversely related to weight, BMI, WC, WHR, and WHtR (all, $P<0.01)$. Among women, no significant correlation was found in RPE with all the independent variables, however, predicted $\mathrm{VO}_{2 \max }$ was inversely related to weight, height, BMI, WC, WHR, and WHtR (all, $P<0.05$ ).

\section{Multiple regression for anthropometric with aerobic fitness in men and women}

The stepwise multiple regressions were performed for weight, BMI, WC, WHR, and WHtR to predict the aerobic fitness in men as the correlation values were above 0.4 . The result demonstrated that WC was a significant predictor for aerobic fitness in both men and women (Table 3). The variance of the predicted $\mathrm{VO}_{2 \max }$ in men was $32.6 \%$ (beta $=-0.571, t(98)=-6.854, P<0.01$ ). WC explained the significant variance in the predicted $\mathrm{VO}_{2 \max }$ in men $\left(F[1,97]=46.971, P<0.01, R^{2}=0.571, R^{2}\right.$ adjusted $\left.=0.319\right)$. This finding could explain that with every $1 \mathrm{~cm}$ increase in WC is associated with a $0.461 \mathrm{~mL} / \mathrm{kg} / \mathrm{min}$ decline in $\mathrm{VO}_{2 \max }$. The $\mathrm{VO}_{2 \max }$ using this model was calculated as follows: $\mathrm{VO}_{2 \max }=$ $76.885-(0.461 \times \mathrm{WC}[\mathrm{cm}])$.

In women, the variance of the predicted $\mathrm{VO}_{2 \max }$ was $33.7 \%$ (beta $=-0.581, t(128)=-8.042, P<0.01)($ Table 3$)$. WC explained a significant amount of variance in the predicted $\mathrm{VO}_{2 \max }$ in wom-

Table 2. Pearson correlations between anthropometric with RPE and predicted $\mathrm{VO}_{2 \max }$

\begin{tabular}{|c|c|c|c|c|c|c|c|c|}
\hline \multirow{3}{*}{ Characteristic } & \multicolumn{4}{|c|}{ Men $(n=99)$} & \multicolumn{4}{|c|}{ Women $(n=129)$} \\
\hline & \multicolumn{2}{|c|}{ RPE } & \multicolumn{2}{|c|}{ Predicted $\mathrm{VO}_{2 \max }$} & \multicolumn{2}{|c|}{ RPE } & \multicolumn{2}{|c|}{ Predicted $\mathrm{VO}_{2 \max }$} \\
\hline & $r$ & $P$-value & $r$ & $P$-value & $r$ & $P$-value & $r$ & $P$-value \\
\hline Weight (kg) & -0.057 & 0.573 & -0.521 & $0.001^{* *}$ & -0.047 & 0.598 & -0.571 & $0.001^{* *}$ \\
\hline Height (cm) & -0.050 & 0.626 & -0.146 & 0.149 & -0.021 & 0.814 & -0.287 & $0.001^{*}$ \\
\hline $\mathrm{BMI}\left(\mathrm{kg} / \mathrm{m}^{2}\right)$ & -0.010 & 0.920 & -0.461 & $0.001^{* *}$ & -0.110 & 0.213 & -0.545 & $0.001^{* *}$ \\
\hline WC $(\mathrm{cm})$ & 0.032 & 0.753 & -0.571 & $0.001^{* *}$ & -0.033 & 0.708 & -0.581 & $0.001^{* *}$ \\
\hline WHR & 0.169 & 0.095 & -0.487 & $0.001^{* *}$ & 0.018 & 0.837 & -0.473 & $0.001^{* *}$ \\
\hline WHtR & 0.072 & 0.481 & -0.516 & $0.001^{* *}$ & -0.040 & 0.650 & -0.545 & $0.001^{* *}$ \\
\hline
\end{tabular}

Comparisons were tested using Pearson correlation coefficient analysis.

$\mathrm{RPE}$, rate perceive exertion; $\mathrm{VO}_{2 \text { max }}$, maximal oxygen consumption; $\mathrm{BMl}$, body mass index; $\mathrm{WC}$, waist circumference; WHR, waist-to-hip ratio; WHtR, waist-to-height ratio.

${ }^{*}$ Correlation is significant at the level of $P<0.05$. ${ }^{*}$ Correlation is significant at the level of $P<0.01$. 
Razak S, et al. - Anthropometry and aerobic fitness

Table 3. Multiple linear regression between anthropometric and predicted $\mathrm{VO}_{2 \max }$ in men and women

\begin{tabular}{|c|c|c|c|c|c|c|c|}
\hline \multirow{2}{*}{ Predicted $\mathrm{VO}_{2 \max }$} & \multicolumn{2}{|c|}{ Unstandardized coefficients } & \multirow{2}{*}{$\begin{array}{c}\text { Standardized coefficients } \\
\beta\end{array}$} & \multirow{2}{*}{$r^{2}$} & \multirow{2}{*}{$P$-value } & \multirow{2}{*}{ Durbin-Watson } & \multirow{2}{*}{ VIF } \\
\hline & B & SEB & & & & & \\
\hline \multicolumn{8}{|l|}{ Men } \\
\hline Constant & 76.885 & 5.821 & -0.571 & 0.326 & $0.001^{* *}$ & 1.478 & 1 \\
\hline WC & -0.461 & 0.067 & & & & & \\
\hline \multicolumn{8}{|l|}{ Women } \\
\hline Constant & 72.745 & 4.239 & -0.581 & 0.337 & $0.001^{* *}$ & 2.006 & 1 \\
\hline WC & -0.440 & 0.055 & & & & & \\
\hline
\end{tabular}

Comparisons were tested using multiple linear regressions.

$\mathrm{VO}_{2 \max }$, maximal oxygen consumption; $\mathrm{SE}$, standard error; VIF, variation inflation factor; $\mathrm{WC}$, waist circumference.

${ }^{*} P<0.01$, significant differences.

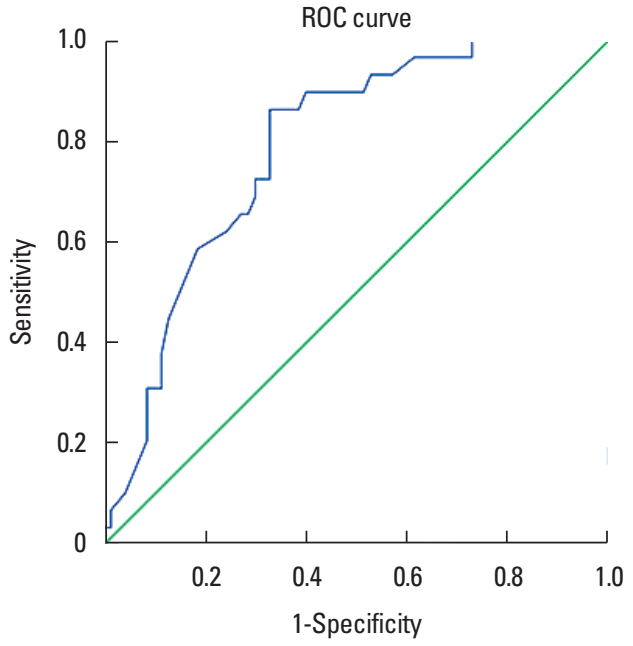

Fig. 1. The receiver operating characteristic (ROC) curve for waist circumference in males. Diagonal segments are produced by ties.

en $\left(F[1,127]=64.681, P<0.01, R^{2}=0.581, R^{2}\right.$ adjusted $\left.=0.332\right)$. Thus, in women, every $1-\mathrm{cm}$ increase in $\mathrm{WC}$ is associated with a $0.44 \mathrm{~mL} / \mathrm{kg} / \mathrm{min}$ unit decline in $\mathrm{VO}_{2 \max }$. The associations between $\mathrm{WC}$ and $\mathrm{VO}_{2 \max }$ in men and women are also significant $(P<0.01)$. The $\mathrm{VO}_{2 \max }$ for women was calculated using this model as follows: $\mathrm{VO}_{2 \max }=72.745-(0.44 \times \mathrm{WC}[\mathrm{cm}])$. There was no analysis carried out for RPE as Pearson correlation results showed no significant association with all the independent variables for both sexes.

\section{ROC curve of WC to predict an aerobic fitness}

The ROC curves analysis was performed to demonstrate the utility of detecting metabolic syndrome risk and heart disease based on aerobic fitness for men (Fig. 1) and women (Fig. 2), respectively. The area under the curve (AUC) for both men (AUC= 78.6; 95\% confidence interval [CI], 0.695-0.877) and women $($ AUC $=83.1 ; 95 \%$ CI, $0.753-0.908)$ were considered high. The

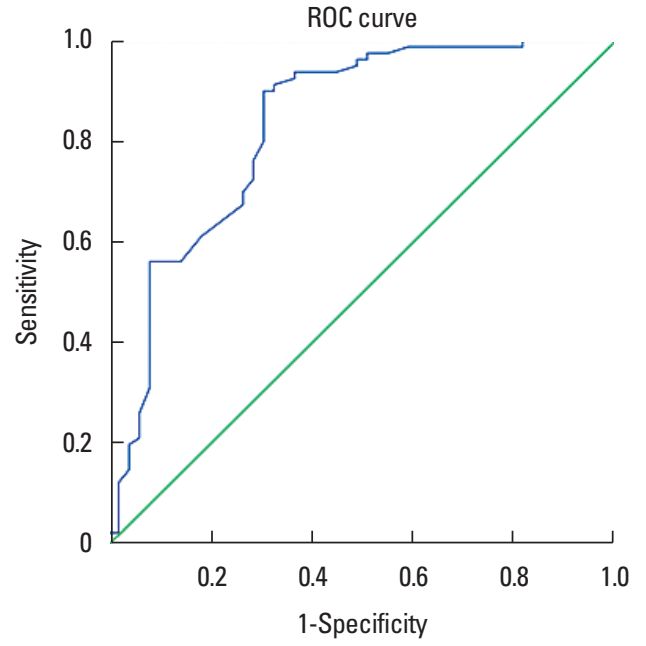

Fig. 2. The receiver operating characteristic (ROC) curve for waist circumference in females. Diagonal segments are produced by ties.

cutoff point of WC in men at optimal sensitivity (86.2\%) and specificity (38.6\%) was $83.75 \mathrm{~cm}$, while, in women, the cutoff point at optimal sensitivity (93.8\%) and specificity (36.7\%) was $81.25 \mathrm{~cm}$.

\section{DISCUSSION}

The analysis of the primary objective of this study showed a few parameters significantly differed between men and women, consistent with a previous study (Fryar et al., 2016). These findings can be attributed to the higher lean mass and bone mass density, as well as visceral fat in men compared to women (He et al., 2018). Although men in the present study had normal WC values, accumulated visceral fat in the abdominal cavity contributed to a large WC in men.

In the current study, the predicted $\mathrm{VO}_{2 \max }$ was associated with 
anthropometric measurements in men and women. This study exhibited a significant inverse correlation between predicted $\mathrm{VO}_{2 \max }$ with the bodyweight that was demonstrated in both genders. These findings are in agreement with an intervention that was performed among young adults in which weight contributed to the improvement of $\mathrm{VO}_{2 \max }$ (Schubert et al., 2016). Among women, it was found that height was negatively associated with $\mathrm{VO}_{2 \max }$, however, this is inconsistent with a previous study that found young men showed that height was positively correlated with aerobic capacity (Siahkouhian, 2009). An individual's height has been associated with heart size; that is, a tall person will have a larger left atrium and ventricle (Siahkouhian, 2009), allowing an enhanced cardiac function to pump blood and oxygen throughout the entire body (Zhang et al., 2018).

The current study also found a strong inverse correlation of WC, WHR, and $\mathrm{WHtR}$ with the predicted $\mathrm{VO}_{2 \max }$ in the men and women. Similar results were obtained in a previous study, in which the predicted $\mathrm{VO}_{2 \max }$ was negatively associated with $\mathrm{WC}, \mathrm{WHtR}$, and WHR among adults (Mondal and Mishra, 2017). Hence, excessive fat deposition in the abdominal area is suggested to increase the burden on cardiac function regardless of sex. The BMI also demonstrated a significant correlation with the predicted $\mathrm{VO}_{2 \max }$ for men and women. A previous study supported that BMI in healthy adults is negatively correlated with the predicted $\mathrm{VO}_{2 \max }$ and that overweight participants presented lower $\mathrm{VO}_{2 \max }$ than those with normal weight (Shah et al., 2016). An increase in BMI is associated with an increase in energy intake, but low energy expenditure and $\mathrm{BMI}$ is strongly associated with fat percentage.

The current finding also suggested that $\mathrm{WC}$ is a strong predictor of aerobic fitness in men and women, and this finding is consistent with previous investigation (Dagan et al., 2013). WC is a direct, easy, inexpensive, and rapid measurement (Tian et al., 2017). WC measurement also is the best indicator of abdominal fat distribution, and high WC will increase the incidence of metabolic risk, type 2 diabetes, and cardiovascular diseases (Siren et al., 2012). Men have been proven to be at a higher risk of these diseases (Tian et al., 2017). Interestingly, one study among the Malaysian population reported that WC was superior to WHR in indicating abdominal obesity (Ahmad et al., 2016). Hence, waist loss in men may be superior to reducing other body compositions to increase their health and physical well-being.

The ROC analysis in the present study indicated that WC is a good diagnostic method to predict cardiovascular disease. This result was consistent with a previous study that also was carried out in the Malaysian population who attended a primary care clinic, where $\mathrm{WC}$ was superior to BMI to predict obesity-related cardiovascular problems, providing the cutoff point of WC were 83$92 \mathrm{~cm}$ and $83-88 \mathrm{~cm}$ in men and women, respectively (Zaher et al., 2009). Another study that was carried out among Indian adults aged 20 years and above found that $\mathrm{WC}$ was a significant predictor for hypertension with cutoff point $>83 \mathrm{~cm}$ in men and $>78$ $\mathrm{cm}$ in women (Midha, 2014), while a study in Japan suggested of $85 \mathrm{~cm}$ and $80 \mathrm{~cm}$ in women to predict diabetes mellitus (Hu et al., 2016). The visceral fat deposition was proven to be more harmful than subcutaneous fat (Shah et al., 2014) due to its negative association with cardiovascular diseases, diabetes and hypertension and is associated with an increase in coronary artery calcium accumulation which may lead to a cardiovascular problem (Shah et al., 2014). This could be due to the excess deposition of fat at the visceral area that may have led to excess accumulation of fat in the liver and muscle, which causes insulin signaling problems (Hardy et al., 2012).

The findings of this study suggested that WC that reflects abdominal obesity is a significant predictor and has a major influence on aerobic fitness and provided the cutoff scores for WC in both Malaysian men and women. Hence, by measuring WC alone, one's health status can be determined, and the risk of cardiovascular disease can be predicted. Generally, this study highlights that a cycle ergometer may be more practical, especially in a clinical setting to measure aerobic fitness which incorporates a nonweight bearing activity. This activity is suitable for the physically inactive individuals where it may prevent the discomfort on the weightbearing joints. Moreover, this test is a submaximal test that was carried out at the same phase throughout the test which has a low risk of withdrawal.

The findings of this study have implications on clinical practices. Firstly, weight loss program not only can focus on weight reduction to have a slimmer body, it also should focus on reducing the abdominal obesity which is the WC measurement that can improve one's overall health. Secondly. this result may not be applicable to younger ( $<18$ years) or older ( $>40$ years) age groups as this study was conducted among individuals aged $18-40$ years. A different result is expected from younger and older groups that warrant further study. One of the limitations of the current study is the use of an estimation method for calculating $\mathrm{VO}_{2 \max }$ rather than directly measuring this parameter. Gas analysis is known to be a gold standard for measuring $\mathrm{VO}_{2 \max }$ during exercise testing, however, this measurement is expensive and requires skilled and trained operators to conduct the test. On the other hand, the study population in this study was between $18-40$ years of healthy but 
physically inactive in which the findings from this study cannot be generalized to other populations.

In conclusion, WC, WHR, WHtR, and BMI were significantly correlated with aerobic fitness among men. In women, weight, BMI, WC, WHR, WHtR, and height were significantly correlated with $\mathrm{VO}_{2 \max }$. WC was the strongest predictor for aerobic fitness in both genders. Thus, reducing fat deposition in the abdominal area can be highlighted in body weight management to enhance a person's health. In addition, awareness on WC measure should be included in patient's education.

\section{CONFLICT OF INTEREST}

No potential conflict of interest relevant to this article was reported.

\section{ACKNOWLEDGMENTS}

The authors received no financial support for this article.

\section{REFERENCES}

Ahmad N, Adam SI, Nawi AM, Hassan MR, Ghazi HF. Abdominal obesity indicators: waist circumference or waist-to-hip ratio in Malaysian adults population. Int J Prev Med 2016;7:82.

Alshamiri MQ Mohd A Habbab F, Al-Qahtani SS, Alghalayini KA, Al-Qat$\tan \mathrm{OM}$, El-Shaer F. Waist-to-height ratio (WHtR) in predicting coronary artery disease compared to body mass index and waist circumference in a single center from Saudi Arabia. Cardiol Res Pract 2020; 2020:4250793.

American College of Sports Medicine. ACSM's guidelines for exercise testing and prescription. Pescatello LS, Arena R, Riebe D, Thompson PD, editors. 9th ed. Lippincott Williams \& Wilkins; 2013. p. 72.

Amirabdollahian F, Haghighatdoost F. Anthropometric indicators of adiposity related to body weight and body shape as cardiometabolic risk predictors in British young adults: superiority of waist-to-height ratio. J Obes 2018;2018:8370304.

Åstrand PO, Ryhming I. A nomogram for calculation of aerobic capacity (physical fitness) from pulse rate during submaximal work. J Appl Physiol 1954;7:218-221.

Badawy MM, Muaidi QI. Cardio respiratory response: validation of new modifications of Bruce protocol for exercise testing and training in elite Saudi triathlon and soccer players. Saudi J Biol Sci 2019;26:105111.

Borg GAV. Psychophysical bases of perceived exertion. Plast Reconstr Surg
1982;14:377-381.

Dagan SS, Segev S, Novikov I, Dankner R. Waist circumference vs body mass index in association with cardiorespiratory fitness in healthy men and women: a cross sectional analysis of 403 subjects. Nutr J 2013; 12:12.

Elsangedy HM, Krinski K, Costa EC, Haile L, Fonteles AI, Timossi LdS, Gregorio da Silva S. The rating of perceived exertion is not different at the ventilatory threshold in sedentary women with different body mass indices. J Exerc Sci Fit 2013;11:102-106.

Fryar C, Gu Q, Ogden C, Flegal K. Anthropometric reference data for children and adults: United States, 2011-2014. Vital Heal Stat 2016;39: $1-46$.

Hardy OT, Czech MP, Corvera S. What causes the insulin resistance underlying obesity? Curr Opin Endocrinol Diabetes Obes 2012;19:81-87.

He X, Li Z, Tang X, Zhang L, Wang L, He Y, Jin T, Yuan D. Age- and sexrelated differences in body composition in healthy subjects aged 18 to 82 years. Medicine (Baltimore) 2018;97:e11152.

Hu H, Kurotani K, Sasaki N, Murakami T, Shimizu C, Shimizu M, Nakagawa T, Honda T, Yamamoto S, Okazaki H, Nagahama S, Uehara A, Yamamoto M, Tomita K, Imai T, Nishihara A, Kochi T, Eguchi M, Miyamoto T, Hori A, Kuwahara K, Akter S, Kashino I, Kabe I, Liu W, Mizoue T, Kunugita N, Dohi S, the Japan Epidemiology Collaboration on Occupational Health Study Group. Optimal waist circumference cut-off points and ability of different metabolic syndrome criteria for predicting diabetes in Japanese men and women: Japan Epidemiology collaboration on occupational health study. BMC Public Health 2016;3:220

Janssen I, Katzmarzyk PT, Ross R. Waist circumference and not body mass index explains obesity-related health risk. Am J Clin Nutr 2004;79:379384.

Lennon OC, Denis RS, Grace N, Blake C. Feasibility, criterion validity and retest reliability of exercise testing using the Astrand-rhyming test protocol with an adaptive ergometer in stroke patients. Disabil Rehabil 2012;34:1149-1156.

Loftin M, Sothern M, Warren B, Udall J. Comparison of $\mathrm{VO}_{2}$ peak during treadmill and cycle ergometry in severely overweight youth. J Sport Sci Med 2004;3:254-260.

Midha T. Cut-off of body mass index and waist circumference to predict hypertension in Indian adults. World J Clin Cases 2014;2:272.

Mondal H, Mishra S. Correlation of waist circumference and waist-toheight ratio with maximal aerobic capacity in young adults. J Heal Res Rev 2017;4:62.

National Health and Morbidity Survey 2019 [Internet]. Shah Alam (Malaysia): National Health and Morbidity Survey; 2019 [cited 2020 July 26]. Available from: http://www.iku.gov.my/images/IKU/Document/ 
REPORT/NHMS2019/Infographic_Booklet_NHMS_2019-English.pdf.

Oueslati F, Berriri A, Boone J, Ahmaidi S. Respiratory muscle strength is decreased after maximal incremental exercise in trained runners and cyclists. Respir Physiol Neurobiol 2018;248:25-30.

Rehman A, Dawood M. Does new public management practices lead to effective public welfare. Turkish Econ Rev 2017;5:150-173.

Schubert MM, Washburn RA, Honas JJ, Lee J, Donnelly JE. Exercise volume and aerobic fitness in young adults: the Midwest Exercise Trial-2. Springerplus 2016;5:183.

Shah H, Prajapathi T, Singh S. Association of body mass index with $\mathrm{VO}_{2 \max }$ in Indian adults. Int J Basic Appl Physiol 2016;5:155-159.

Shah RV, Murthy VL, Abbasi SA, Blankstein R, Kwong RY, Goldfine AB, Jerosch-Herold M, Lima JAC, Ding J, Allison MA. Visceral adiposity and the risk of metabolic syndrome across body mass index: the MESA study. JACC Cardiovasc Imaging 2014;7:1221-1235.

Siahkouhian M. Impact of height on the prediction of maximum oxygen consumption in active young men. J Appl Sci 2009;9:2340-2343.

Siren R, Eriksson JG, Vanhanen H. Waist circumference a good indicator of future risk for type 2 diabetes and cardiovascular disease. BMC Public Health 2012;12:631.

Tian X, Xu X, Zhang K, Wang H. Gender difference of metabolic syndrome and its association with dietary diversity at different ages. Oncotarget 2017;8:73568-73578.

Tran NTT, Blizzard CL, Luong KN, Truong NLV, Tran BQ, Otahal P, Nelson M, Magnussen C, Gall S, Bui TV, Srikanth V, Au TB, Ha ST, Phung HN, Tran MH, Callisaya M. The importance of waist circumference and body mass index in cross-sectional relationships with risk of cardiovascular disease in Vietnam. PLoS One 2018;13:e0198202.

Vancampfort D, De Herdt A, Vanderlinden J, Lannoo M, Adriaens A, De Hert M, Stubbs B, Soundy A, Probst M. The functional exercise capacity and its correlates in obese treatment-seeking people with binge eating disorder: an exploratory study. Disabil Rehabil 2015;37:777-782.

Zaher ZM, Zambari R, Pheng CS, Muruga V, Ng B, Appannah G, Onn LT. Optimal cut-off levels to define obesity: body mass index and waist circumference, and their relationship to cardiovascular disease, dyslipidaemia, hypertension and diabetes in Malaysia. Asia Pac J Clin Nutr 2009;18:209-216.

Zhang Y, Qi L, Xu L, Sun X, Liu W, Zhou S, van de Vosse F, Greenwald SE. Effects of exercise modalities on central hemodynamics, arterial stiffness and cardiac function in cardiovascular disease: systematic review and meta-analysis of randomized controlled trials. PLoS One 2018;13:e0200829. 